\section{Studien, diagnostische}

R.-D. Hilgers ${ }^{1}$, N. Heussen ${ }^{1}$ und S. Stanzel ${ }^{2}$

${ }^{1}$ Institut für Medizinische Statistik, Universitätsklinikum der RWTH Aachen, Aachen, Deutschland

${ }^{2}$ DKFZ HEidelberg, Heidelberg, Deutschland

Englischer Begriff diagnostic studies

Definition Diagnostische Studien sind Spezialformen klinischer bzw. epidemiologischer Studien mit der Zielsetzung der Bewertung unterschiedlicher Aspekte eines diagnostischen Prozesses.

Beschreibung Je nach Zielsetzung sind die in der folgenden Tabelle aufgelisteten Studientypen zu verwenden.

\begin{tabular}{|l|l|l|}
\hline $\begin{array}{l}\text { Allgemeine } \\
\text { Studientypen }\end{array}$ & Fragestellung & $\begin{array}{l}\text { Mögliche } \\
\text { Studientypen }\end{array}$ \\
\hline Klinische Studien & $\begin{array}{l}\text { Diagnostische } \\
\text { Accuracy }\end{array}$ & $\begin{array}{l}\text { Querschnittstudie } \\
\text { Fall-Kontroll-Studie } \\
\text { Stichprobensammlung } \\
\text { basierend auf } \\
\text { Testresultaten }\end{array}$ \\
& & $\begin{array}{l}\text { Befragungen in } \\
\text { indizierten } \\
\text { Populationen }\end{array}$ \\
\hline & & $\begin{array}{l}\text { Randomisierte } \\
\text { kontrollierte klinische } \\
\text { Studie }\end{array}$ \\
\hline & $\begin{array}{l}\text { Wertigkeit eines } \\
\text { diagnostischen Tests } \\
\text { für die Prognose oder }\end{array}$ & Kohortenstudie \\
\hline
\end{tabular}

(Fortsetzung)

\begin{tabular}{|c|c|c|}
\hline $\begin{array}{l}\text { Allgemeine } \\
\text { Studientypen }\end{array}$ & Fragestellung & $\begin{array}{l}\text { Mögliche } \\
\text { Studientypen }\end{array}$ \\
\hline \multirow{10}{*}{$\begin{array}{l}\text { Zusammenfassende } \\
\text { Bewertung }\end{array}$} & \multirow{2}{*}{$\begin{array}{l}\text { das } \\
\text { Behandlungsregime }\end{array}$} & Fall-Kontroll-Studie \\
\hline & & $\begin{array}{l}\text { Vor-Nach- } \\
\text { Vergleichsstudie }\end{array}$ \\
\hline & \multirow{3}{*}{$\begin{array}{l}\text { Zusammenfassung der } \\
\text { Ergebnisse aus } \\
\text { mehreren Studien }\end{array}$} & Systematischer \\
\hline & & Review \\
\hline & & Meta-Analyse \\
\hline & \multirow{2}{*}{$\begin{array}{l}\text { Bestimmung der } \\
\text { kosteneffektivsten } \\
\text { diagnostischen } \\
\text { Strategie }\end{array}$} & $\begin{array}{l}\text { Klinische } \\
\text { Entscheidungsanalyse }\end{array}$ \\
\hline & & $\begin{array}{l}\text { Kosten-Effektivitäts- } \\
\text { Analyse }\end{array}$ \\
\hline & \multirow[t]{3}{*}{$\begin{array}{l}\text { Übertragung der } \\
\text { Ergebnisse in die } \\
\text { Praxis }\end{array}$} & $\begin{array}{l}\text { Integration der } \\
\text { Ergebnisse aus den } \\
\text { vorangehenden } \\
\text { Studientypen }\end{array}$ \\
\hline & & $\begin{array}{l}\text { Experten-Konsensus- } \\
\text { Konferenz }\end{array}$ \\
\hline & & $\begin{array}{l}\text { Entwicklung von } \\
\text { Leitlinien }\end{array}$ \\
\hline \multirow[t]{2}{*}{$\begin{array}{l}\text { Nutzung der } \\
\text { Ergebnisse in der } \\
\text { Praxis }\end{array}$} & \multirow[t]{2}{*}{ ICT-Studien } & $\begin{array}{l}\text { Überprüfung der } \\
\text { Lösung des } \\
\text { diagnostischen } \\
\text { Problems }\end{array}$ \\
\hline & & $\begin{array}{l}\text { Evaluation der } \\
\text { Implementierung in die } \\
\text { Praxis }\end{array}$ \\
\hline
\end{tabular}

\section{Literatur}

Knottnerus JA, van Weel C, JWM M (2002) Evidence base of clinical diagnosis - evaluation of diagnosis procedures. BMJ 23:477-480 